\title{
Sarcopenia is associated with the presence of nonalcoholic fatty liver disease in Zhejiang Province, China: a cross-sectional observational study
}

Yu-Ming Wang ${ }^{1,2+}$, Ke-Fu Zhu ${ }^{3 \dagger}$, Wen-Jing Zhou ${ }^{1,2}$, Qin Zhang ${ }^{1,2}$, Dan-Feng Deng ${ }^{1,2}$, Yi-Chen Yang ${ }^{1,2}$, Wen-Wen Lu $u^{1,2}$, Jia Xu $\mathrm{u}^{2,4}$ and Yun-Mei Yang ${ }^{1,2^{*}}$ (D)

\begin{abstract}
Background: Currently, both non-alcoholic fatty liver disease (NAFLD) and sarcopenia have attracted extensive attention in public health. However, the relationship between NAFLD and sarcopenia remains unclear. This study aimed to clarify the sex-specific association between sarcopenia and NAFLD according to the Asian Working Group for Sarcopenia (AWGS).

Methods: Dual-energy X-ray absorptiometry (DXA) and hepatic ultrasonography were measured in 578 participants (92 men and 486 women) during their annual health examinations. Multivariate logistic regression models were used to explore the association between NAFLD and sarcopenia with its two components.

Results: A total of 154 participants (30 men and 124 women) had NAFLD. The prevalence of sarcopenia was higher among the participants with NAFLD than among those without NAFLD (men: $20.0 \%$ vs. 9.7\%, $P=0.295$, women: $15.3 \%$ vs. $8.0 \%, P=0.019$ ). Low muscle mass (LMM) was independently associated with NAFLD in both men and women (men: odds ratio [OR], 2.88; 95\% confidence interval [CI] 1.52-5.46; women: OR, 2.08; 95\% Cl 1.63-2.67). However, low muscle strength (LMS) was independently associated with NAFLD only in male participants, with an OR of 1.15 (95\% Cl 1.02-1.28).
\end{abstract}

Conclusion: The occurrence of sarcopenia was associated with a higher risk of NAFLD, especially in men, as demonstrated by lower muscle mass and lower muscle strength.

Keywords: Muscle mass, Muscle strength, Non-alcoholic fatty liver disease, Sarcopenia

\footnotetext{
* Correspondence: 1194070@zju.edu.cn

${ }^{\dagger}$ Yu-Ming Wang and Ke-Fu Zhu contributed equally to this work.

'Department of Geriatrics, the First Affiliated Hospital, School of Medicine,

Zhejiang University, Hangzhou 310003, Zhejiang, China

${ }^{2}$ Zhejiang Provincial Key Laboratory for Diagnosis and Treatment of Aging and Physic-chemical Injury Diseases, the First Affiliated Hospital, School of Medicine, Zhejiang University, Hangzhou 310003, Zhejiang, China

Full list of author information is available at the end of the article
}

C C The Author(s). 2021 Open Access This article is licensed under a Creative Commons Attribution 4.0 International License, which permits use, sharing, adaptation, distribution and reproduction in any medium or format, as long as you give appropriate credit to the original author(s) and the source, provide a link to the Creative Commons licence, and indicate if changes were made. The images or other third party material in this article are included in the article's Creative Commons licence, unless indicated otherwise in a credit line to the material. If material is not included in the article's Creative Commons licence and your intended use is not permitted by statutory regulation or exceeds the permitted use, you will need to obtain permission directly from the copyright holder. To view a copy of this licence, visit http://creativecommons.org/licenses/by/4.0/ The Creative Commons Public Domain Dedication waiver (http://creativecommons.org/publicdomain/zero/1.0/) applies to the data made available in this article, unless otherwise stated in a credit line to the data. 


\section{Background}

Non-alcoholic fatty liver disease (NAFLD) is currently the leading cause of chronic liver disease worldwide, ranging from simple steatosis, non-alcoholic steatohepatitis (NASH), and advanced fibrosis to end-stage liver diseases, such as cirrhosis and hepatocellular carcinoma [1]. The total NAFLD population in 2015 was estimated at 83.1 million cases with a prevalence rate of $30.0 \%$ among the population aged $\geq 15$ years and will increase to $33.5 \%$ by 2030 [2]. NAFLD has been proven to be closely associated with systemic diseases and has attracted extensive attention in public health [3]. The biological mechanisms, such as peripheral resistance to insulin, dyslipidaemia, and the activation of inflammatory pathways associated with NAFLD, are relevant to these systemic diseases [4].

Sarcopenia is a multifactorial geriatric syndrome with the overall concept of skeletal muscle failure or insufficiency. Skeletal muscle, as an active endocrine organ responsible for insulin-mediated glucose disposal, plays a significant role in glucose homeostasis, insulin resistance and inflammation [5]. Meanwhile, sarcopenia is associated with a sedentary lifestyle, which increases the risk of obesity, metabolic syndrome and NAFLD [6]. It has been reported that up to $60 \%$ of patients with end-stage liver disease have sarcopenia. The presence of NASH in those patients was associated with a 6-fold increased risk of sarcopenic obesity $[7,8]$. Thus, sarcopenia shares the common risk factors that contribute to NAFLD and have a plausible association with NAFLD.

Until now, all consensuses have agreed on two crucial components of the sarcopenia definition: sarcopenia involves both structural damage (low muscle mass [LMM]) and impaired function (low muscle strength [LMS]) [9]. Several studies have shown that sarcopenia, including its two crucial components (LMM and LMS), is associated with the prevalence of NAFLD. Kim et al. found that skeletal muscle mass was positively correlated with the occurrence of NAFLD and negatively correlated with the resolution of existing NAFLD [10]. Another study showed that men and women with NAFLD had 7.3 and $7.9 \%$ lower handgrip strength (HGS) than controls in older adults [11]. Since the trajectories of muscle mass and muscle strength decline during ageing do not overlap and muscle strength declines much more rapidly than muscle mass, it is essential to illustrate the association between NAFLD and sarcopenia with its two components at the same time $[12,13]$. Furthermore, almost all clinical studies were conducted in Korean populations, and few were diagnosed according to the Asian Working Group for Sarcopenia (AWGS) consensus guidelines. Therefore, this study aimed to investigate the independent association of the two components of sarcopenia with NAFLD, stratified by sex, in the aged
Chinese population according to the AWGS. In addition, we tried to assess which component could better predict NAFLD prevalence in different sex groups.

\section{Methods \\ Study population}

This is a cross-sectional, observational study of 578 senior hospital staff (92 men and 486 women) who attended annual health examinations at the First Affiliated Hospital, School of Medicine, Zhejiang University, between January 2019 and December 2019. Participants who had full records of personal health history, anthropometric and biochemical data and hepatic ultrasonography results were initially enrolled. Participants who had cancer, viral/druginduced/autoimmune liver diseases, severe cardiopulmonary disorders, renal dysfunction, history of organ transplant, physical or cognitive impairment, or excessive alcohol consumption (males $>140 \mathrm{~g} /$ week or females $>70$ g/week) were excluded [14]. This study was approved by the Ethics Committee of The First Affiliated Hospital, School of Medicine, Zhejiang University, in accordance with the Helsinki Declaration. All participants gave written informed consent before participation.

\section{Laboratory measurements}

All participants underwent a thorough physical examination. Height $(\mathrm{cm})$ and weight $(\mathrm{kg})$ were measured using standardized protocols while the participants were dressed in light clothing without shoes. Body mass index (BMI) $\left(\mathrm{kg} / \mathrm{m}^{2}\right)$ was calculated according to the following formula: $\mathrm{BMI}=$ weight $(\mathrm{kg}) /$ height $(\mathrm{m})^{2}$. According to Asia-Pacific criteria, general obesity was defined as BMI $\geq 25 \mathrm{~kg} / \mathrm{m}^{2}$. After an overnight fast of $\geq 8 \mathrm{~h}$, blood samples were obtained from the peripheral vein of each participant. All laboratory measurements, including liver function tests (alanine aminotransferase [ALT], aspartate aminotransferase [AST], and gamma-glutamyltransferase [GGT]), lipid profile (triglyceride [TG], total cholesterol [TC], high-density lipoprotein cholesterol [HDL-C], and low-density lipoprotein cholesterol [LDL-C]), fasting plasma glucose (FPG), albumin (ALB), creatinine (Cr) and uric acid (UA), were measured by a Hitachi 7600 autoanalyser (Hitachi, Tokyo, Japan) using standard protocols.

\section{Diagnosis of sarcopenia and relative parameters}

Participants who had completed dual-energy X-ray absorptiometry (DXA) tests for HGS and $4 \mathrm{~m}$ walking speed were selected. A gait speed (GS) $<0.8 \mathrm{~m} / \mathrm{s}$ was defined as low GS, as recommended by the AWGS. Both the left and right HGSs were measured with a Jamar Hydraulic Hand Dynamometer (Jamar Hydraulic Hand Dynamometer Model 5030 J1; Sammons Preston, Bolingbrook, IL, USA) three times, and the maximum value was used, according 
to the recommendations of the American Society of Hand Therapists (ASHT). LMS was defined as $<26.0 \mathrm{~kg}$ in men and $<18.0 \mathrm{~kg}$ in women, as recommended by the AWGS. Skeletal muscle mass was estimated by the skeletal muscle index (SMI) using the appendicular skeletal muscle mass (ASM) divided by body height squared $\left(\mathrm{kg} / \mathrm{m}^{2}\right)$, which was measured by DXA using a Hologic Discovery ${ }^{\text {Tm }}$ device (Hologic, Waltham, MA, USA). LMM was defined as $\leq 7.0$ $\mathrm{kg} / \mathrm{m}^{2}$ in men and $\leq 5.4 \mathrm{~kg} / \mathrm{m}^{2}$ in women as recommended by the AWGS. Sarcopenia was diagnosed according to the criteria of the AWGS [15] (Fig. 1). Sarcopenic obesity was defined as the presence of both sarcopenia and obesity.

\section{Diagnosis of NAFLD}

NAFLD was determined based on the result of a hepatic ultrasound examination following the exclusion of alcohol consumption and viral or autoimmune liver disease. Hepatic ultrasonography (US; Acuson Sequoia 512, Siemens, Mountain View, CA, USA) was carried out by experienced ultrasonographers. The ultrasonographers were blinded to study design and clinical data. The criteria for the ultrasonic diagnosis of fatty liver were based on those recommended by the Chinese Liver Disease Association [16].

\section{Statistical analysis}

Continuous variables are presented as the mean \pm standard deviation (SD), and categorical variables are presented as the frequency (percentage). The statistical significance of the differences in clinical and biochemical values between participants with and without NAFLD was analysed by sex using Student's t-test for continuous variables and the chi-squared test for categorical variables. The Pearson correlation coefficient was calculated to assess the associations between muscle mass or muscle strength and the relative parameters of NAFLD. Multivariate logistic regression models were performed to calculate the adjusted OR and 95\% CI to explore the associations of NAFLD with muscle mass and muscle strength. The following factors were considered independent variables for multivariate logistic regression analysis: Model 1: age and weight; Model 2: model 1 covariates plus BMI, TG, and ALT. A receiver operating characteristic (ROC) curve of muscle mass was developed to predict the presence of NAFLD in both men and women. All calculations were performed using IBM SPSS Statistics ver. 22.0 (IBM Co., Armonk, NY, USA), and the associated results were plotted using GraphPad Prism 6 (GraphPad, San Diego, California, USA). Two-sided $P$-values $<0.05$ were considered statistically significant.

\section{Result}

Basic laboratory and clinical characteristics

The basic laboratory and clinical characteristics of the 578 participants (92 men and 486 women) with and without NAFLD enrolled in this study are shown in Table 1. Thirty (32.6\%) male and 124 (25.5\%) female participants had NAFLD. Among the men, the participants with NAFLD were younger and had higher body weight, BMI, serum TG, and ALT than those without

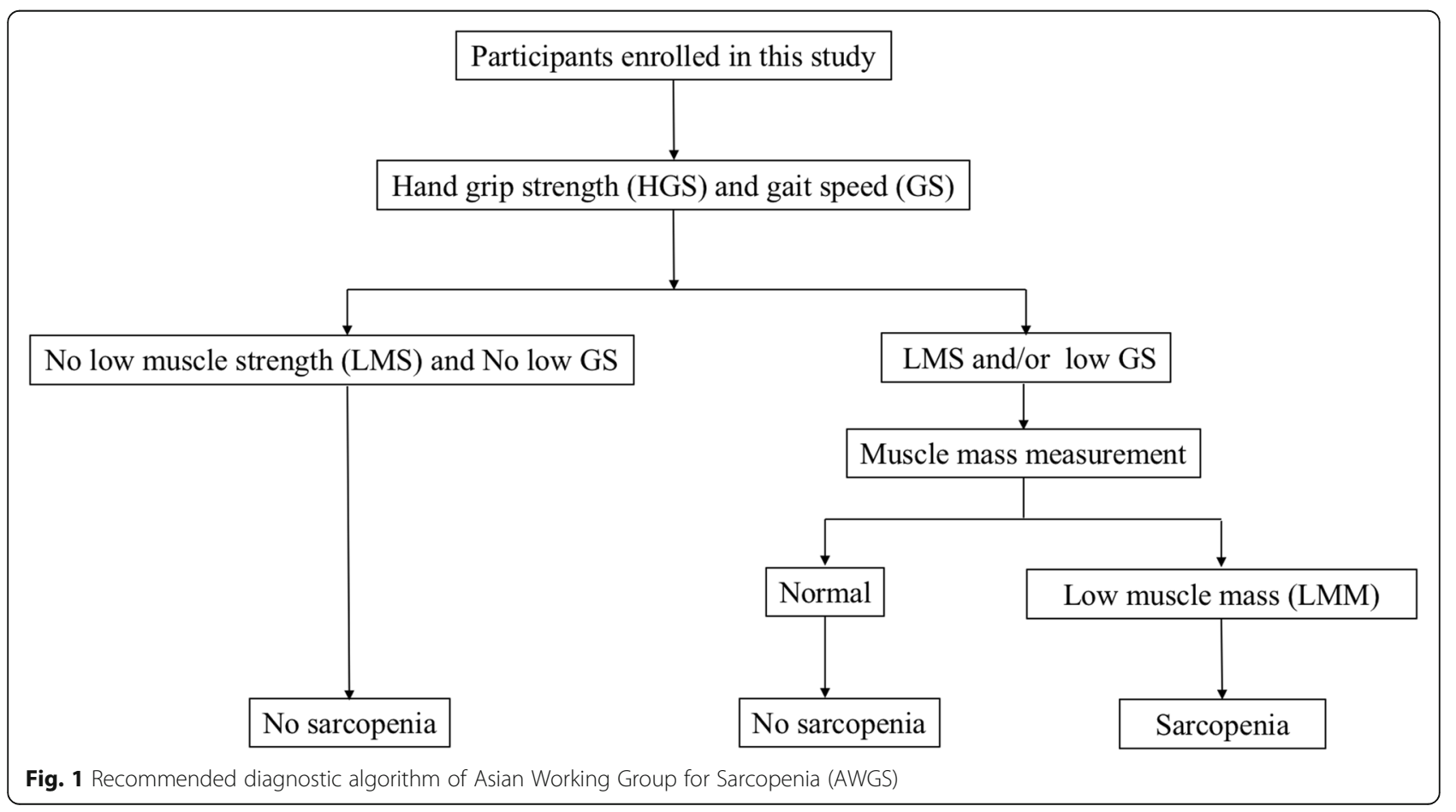


Table 1 Baseline characteristics of the study participants, categorized according to the presence or absence of NAFLD

\begin{tabular}{|c|c|c|c|c|c|c|}
\hline \multirow[b]{2}{*}{ Variable } & \multicolumn{3}{|l|}{$\operatorname{Men}(n=92)$} & \multicolumn{3}{|l|}{ Women $(n=486)$} \\
\hline & $\begin{array}{l}\text { Without NAFLD } \\
(n=62)\end{array}$ & $\begin{array}{l}\text { With NAFLD } \\
(n=30)\end{array}$ & $P$ & $\begin{array}{l}\text { Without NAFLD } \\
(n=362)\end{array}$ & $\begin{array}{l}\text { With NAFLD } \\
(n=124)\end{array}$ & $P$ \\
\hline Ages (years) & $72.9 \pm 9.2$ & $68.9 \pm 5.8$ & 0.029 & $62.9 \pm 12.1$ & $67.5 \pm 10.1$ & $<0.001$ \\
\hline Weight (kg) & $67.3 \pm 9.6$ & $75.3 \pm 12.2$ & $<0.001$ & $55.8 \pm 7.0$ & $61.5 \pm 8.3$ & $<0.001$ \\
\hline Height (cm) & $170.7 \pm 6.1$ & $169.0 \pm 6.4$ & 0.239 & $157.3 \pm 9.4$ & $157.7 \pm 4.9$ & 0.587 \\
\hline Body mass index $\left(\mathrm{kg} / \mathrm{m}^{2}\right)$ & $23.6 \pm 3.0$ & $25.7 \pm 3.3$ & 0.002 & $22.5 \pm 2.6$ & $24.7 \pm 2.8$ & $<0.001$ \\
\hline TC (mmol/L) & $4.4 \pm 0.9$ & $4.6 \pm 0.9$ & 0.312 & $5.0 \pm 0.9$ & $5.1 \pm 1.1$ & 0.711 \\
\hline TG (mmol/L) & $0.8 \pm 0.5$ & $1.8 \pm 0.7$ & $<0.001$ & $1.2 \pm 0.7$ & $1.6 \pm 1.0$ & $<0.001$ \\
\hline HDL-C (mmol/L) & $1.3 \pm 0.3$ & $1.2 \pm 0.4$ & 0.065 & $1.5 \pm 0.4$ & $1.3 \pm 0.3$ & $<0.001$ \\
\hline LDL-C (mmol/L) & $2.5 \pm 0.7$ & $2.6 \pm 0.8$ & 0.426 & $2.8 \pm 0.7$ & $2.9 \pm 0.9$ & 0.321 \\
\hline AST (IU/L) & $23.0 \pm 3.1$ & $23.6 \pm 4.4$ & 0.665 & $21.3 \pm 6.0$ & $26.1 \pm 7.2$ & 0.003 \\
\hline ALT (IU/L) & $19.3 \pm 7.4$ & $25.5 \pm 14.0$ & 0.007 & $17.6 \pm 10.2$ & $24.5 \pm 14.6$ & $<0.001$ \\
\hline GGT (IU/L & $28.0 \pm 17.6$ & $29.5 \pm 13.7$ & 0.696 & $21.2 \pm 16.8$ & $28.4 \pm 18.4$ & $<0.001$ \\
\hline $\mathrm{ALB}(\mathrm{g} / \mathrm{L})$ & $46.6 \pm 2.4$ & $47.6 \pm 2.0$ & 0.061 & $46.9 \pm 2.4$ & $47.9 \pm 2.5$ & $<0.001$ \\
\hline $\mathrm{Cr}$ (umol/L) & $89.1 \pm 15.3$ & $87.2 \pm 12.3$ & 0.555 & $64.9 \pm 11.0$ & $64.6 \pm 11.1$ & 0.789 \\
\hline FPG (mmol/L) & $5.6 \pm 1.4$ & $5.9 \pm 1.1$ & 0.236 & $5.2 \pm 1.0$ & $5.8 \pm 1.2$ & $<0.001$ \\
\hline UA ( $\mu \mathrm{mol} / \mathrm{L})$ & $357.5 \pm 84.2$ & $371.0 \pm 77.5$ & 0.461 & $267.3 \pm 63.4$ & $305.4 \pm 71.0$ & $<0.001$ \\
\hline Gait speed (m/s) & $1.3 \pm 0.3$ & $1.1 \pm 0.3$ & 0.008 & $1.2 \pm 0.2$ & $1.1 \pm 0.3$ & 0.008 \\
\hline Muscle strength (kg) & $37.6 \pm 9.7$ & $35.8 \pm 8.6$ & 0.404 & $24.5 \pm 5.1$ & $23.4 \pm 5.8$ & 0.053 \\
\hline Muscle mass $\left(\mathrm{kg} / \mathrm{m}^{2}\right)$ & $7.2 \pm 1.3$ & $6.6 \pm 1.2$ & 0.032 & $5.6 \pm 1.1$ & $5.23 \pm 1.3$ & 0.011 \\
\hline Sarcopenia & 6/62 (9.7\%) & 6/30 (20.0\%) & 0.295 & 29/362 (8.0\%) & 19/124 (15.3\%) & 0.019 \\
\hline Sarcopenic obesity & $2 / 62(3.2 \%)$ & $3 / 30(10.0 \%)$ & 0.394 & $3 / 362(0.8 \%)$ & $4 / 124(3.2 \%)$ & 0.134 \\
\hline
\end{tabular}

Values are presented as mean \pm standard deviation, unless otherwise specified. Bold numbers indicate statistically significant values. NAFLD non-alcoholic fatty liver disease, BMI body mass index, TC total cholesterol, TG triglyceride, HDL-C high-density lipoprotein cholesterol, $L D L-C$ low-density lipoprotein cholesterol, $A S T$ aspartate aminotransferase, $A L T$ alanine aminotransferase, GGT gamma-glutamyl transferase, $A L B$ albumin, $C r$ creatinine, $F P G$ fasting plasma glucose, UA uric acid

NAFLD, but there were no differences in height, serum TC, HDL-C, LDL-C, AST, GGT, ALB, Cr, FPG or UA between the two groups. Among the women, the participants with NAFLD were older and had higher weight, BMI, TG, serum AST, ALT, GGT, ALB, FPG, and UA and lower HDL-C than those without NAFLD. However, there were no differences in height, serum TC, LDL-C or Cr between the two groups. As illustrated in Table 1, the levels of GS were lower in patients with NAFLD than in NAFLD-free participants in both men and women (men: $1.1 \pm 0.3$ vs. $1.3 \pm 0.3 \mathrm{~kg} / \mathrm{m}^{2}, P=0.008$; women: $1.1 \pm 0.3$ vs. $\left.1.2 \pm 0.2 \mathrm{~kg} / \mathrm{m}^{2}, P=0.008\right)$. The levels of muscle mass were also significantly lower in patients with NAFLD than in NAFLD-free participants (men: $6.6 \pm 1.2$ vs. $7.2 \pm 1.3 \mathrm{~kg} / \mathrm{m}^{2}, P=0.032$; women: $5.2 \pm 1.3$ vs. $\left.5.6 \pm 1.1 \mathrm{~kg} / \mathrm{m}^{2}, P=0.011\right)$. However, there was no significant difference in the levels of HGS between the two groups (men: $35.8 \pm 8.6$ vs. $37.6 \pm 9.7, P=$ 0.404 , women: $23.4 \pm 5.8$ vs. $24.5 \pm 5.1 \mathrm{~kg}, P=0.053$ ). The prevalence of sarcopenia was higher among the participants with NAFLD (men: $20.0 \%$ vs. 9.7\%, $P=0.295$, women: $15.3 \%$ vs. $8.0 \%, P=0.019)$. Moreover, the prevalence of sarcopenic obesity was also higher in the NAFL
D group in both men and women, but the difference was not significant (men: $10.0 \%$ vs. $3.2 \%, P=0.394$, women: $3.2 \%$ vs. $0.8 \%, P=0.134)$.

\section{Associations of muscle mass and anthropometric and biochemical variables of NAFLD}

We performed Pearson correlation analysis to determine the correlations between muscle mass and anthropometric and biochemical variables of NAFLD. The correlation analyses between anthropometric and biochemical variables and muscle mass in participants are shown in Table 2. We found that muscle mass was positively correlated with body weight $(r=0.292, P=0.005)$, BMI $(r=0.291, P=0.005)$, ALB $(r=0.232, P=0.026)$ and HGS $(r=0.315, P=0.002)$ but negatively correlated with age $(r=-0.244, P=0.019)$ and FPG $(r=-0.251, P=0.016)$ among male participants. Meanwhile, muscle mass was positively correlated with age $(r=0.111, P=0.015)$, body weight $(r=0.295, P<0.001)$, BMI $(r=0.326, P<0.001)$, serum ALT $(r=0.139, P=0.002), \mathrm{Cr}(r=0.193, P<0.001)$, UA $(r=0.142, P=0.002)$, and FPG $(r=0.111, P=0.015)$ among female participants (Table 2). 
Table 2 Pearson correlation coefficients between muscle mass and patient characteristics at baseline by gender (for men and women)

\begin{tabular}{|c|c|c|c|c|}
\hline \multirow[t]{3}{*}{ Variable } & \multicolumn{4}{|c|}{ Muscle mass $\left(\mathrm{kg} / \mathrm{m}^{2}\right)$} \\
\hline & \multicolumn{2}{|c|}{ men } & \multicolumn{2}{|l|}{ women } \\
\hline & $r$ & $P$ & $r$ & $P$ \\
\hline Age (years) & -0.244 & 0.019 & 0.111 & 0.015 \\
\hline Body weight (kg) & 0.292 & 0.005 & 0.295 & $<0.001$ \\
\hline Height (cm) & 0.098 & 0.355 & -0.078 & 0.088 \\
\hline BMI $\left(\mathrm{kg} / \mathrm{m}^{2}\right)$ & 0.291 & 0.005 & 0.326 & $<0.001$ \\
\hline $\mathrm{ALT}(\mathrm{IU} / \mathrm{L})$ & 0.093 & 0.378 & 0.139 & 0.002 \\
\hline AST (IU/L) & 0.243 & 0.167 & 0.177 & 0.101 \\
\hline TG (mmol/L) & -0.025 & 0.815 & -0.01 & 0.834 \\
\hline $\mathrm{TC}(\mathrm{mmol} / \mathrm{L})$ & -0.108 & 0.304 & -0.055 & 0.232 \\
\hline $\operatorname{ALB}(\mathrm{g} / \mathrm{L})$ & 0.232 & 0.026 & -0.003 & 0.943 \\
\hline $\mathrm{Cr}(\mu \mathrm{mol} / \mathrm{L})$ & 0.101 & 0.339 & 0.193 & $<0.001$ \\
\hline UA $(\mu \mathrm{mol} / \mathrm{L})$ & 0.179 & 0.087 & 0.142 & 0.002 \\
\hline $\mathrm{FPG}(\mathrm{mmol} / \mathrm{L})$ & -0.251 & 0.016 & 0.111 & 0.015 \\
\hline Gait speed (m/s) & 0.153 & 0.145 & -0.026 & 0.568 \\
\hline Muscle strength $(\mathrm{kg})$ & 0.315 & 0.002 & -0.041 & 0.364 \\
\hline
\end{tabular}

\section{Associations of HGS and anthropometric and biochemical variables of NAFLD}

We found that HGS was positively correlated with body weight $(r=0.255, P=0.014)$, height $(r=0.51, P<0.001)$, GS $(r=0.408, P<0.001)$ and muscle mass $(r=0.315, P=$ $0.002)$ but negatively correlated with age $(r=-0.504, P<$ $0.001)$ among male participants. HGS was positively correlated with body weight $(r=0.206, P<0.001)$, GS $(r=$ $0.538, P<0.001)$ and height $(r=0.315, P<0.001)$ but negatively correlated with age $(r=-0.533, P<0.001)$, serum AST $(r=-0.391, P<0.001)$, TG $(r=-0.144, P=$ $0.002)$, UA $(r=-0.193, P<0.001)$, and FPG $(r=-0.177$, $P<0.001)$ among female participants (Table 3$)$.

\section{Independent impact of muscle mass and muscle strength} on the presence of NAFLD

A logistic regression model was conducted to evaluate the sex-specific relationship between the components of sarcopenia (LMM or LMS) and NAFLD risk (Models 12, Table 4). The relationship between LMM and NAFLD was statistically significant in both models. In Model 1, the ORs with 95\% CIs for NAFLD were 2.91 (95\% CI $1.58-5.35)$ and 1.89 (95\% CI 1.51-2.38) in men and women, respectively. Furthermore, the fully adjusted model (Model 2) showed that LMM was still associated with an increased risk of NAFLD, with ORs of 2.88 (95\% CI 1.52-5.46) in men and 2.08 (95\% CI 1.63-2.67) in women. Participants with LMS showed significantly high odds of NAFLD, with ORs of 1.15 (95\% CI, 1.04-1.26) and 1.15 (95\% CI 1.02-1.28) in men after adjusting in
Table 3 Pearson correlation coefficients between muscle strength and patient characteristics at baseline by gender (for men and women)

\begin{tabular}{|c|c|c|c|c|}
\hline \multirow[t]{3}{*}{ Variable } & \multicolumn{4}{|c|}{ Muscle strength (kg) } \\
\hline & \multicolumn{2}{|l|}{ men } & \multicolumn{2}{|l|}{ women } \\
\hline & $r$ & $P$ & $r$ & $P$ \\
\hline Age (years) & -0.504 & $<0.001$ & -0.533 & $<0.001$ \\
\hline Body weight (kg) & 0.255 & 0.014 & 0.206 & $<0.001$ \\
\hline Height (cm) & 0.51 & $<0.001$ & 0.315 & $<0.001$ \\
\hline BMI $\left(\mathrm{kg} / \mathrm{m}^{2}\right)$ & 0.02 & 0.848 & -0.018 & 0.692 \\
\hline ALT (IU/L) & 0.062 & 0.554 & -0.065 & 0.156 \\
\hline AST (IU/L) & 0.08 & 0.651 & -0.391 & $<0.001$ \\
\hline $\mathrm{TG}(\mathrm{mmol} / \mathrm{L})$ & 0.15 & 0.152 & -0.144 & 0.002 \\
\hline $\mathrm{TC}(\mathrm{mmol} / \mathrm{L})$ & 0.019 & 0.854 & 0.011 & 0.804 \\
\hline $\operatorname{ALB}(g / L)$ & 0.184 & 0.079 & 0.142 & 0.002 \\
\hline $\mathrm{Cr}(\mu \mathrm{mol} / \mathrm{L})$ & 0.013 & 0.901 & -0.155 & 0.001 \\
\hline UA ( $\mu \mathrm{mol} / \mathrm{L})$ & 0.059 & 0.578 & -0.193 & $<0.001$ \\
\hline $\mathrm{FPG}(\mathrm{mmol} / \mathrm{L})$ & -0.195 & 0.062 & -0.177 & $<0.001$ \\
\hline Gait speed (m/s) & 0.408 & $<0.001$ & 0.538 & $<0.001$ \\
\hline Muscle mass $\left(\mathrm{kg} / \mathrm{m}^{2}\right)$ & 0.315 & 0.002 & -0.041 & 0.364 \\
\hline
\end{tabular}

Model 1 and Model 2, respectively. However, there was no statistical significance in muscle strength in women after adjusting in Model 1 and Model 2, with ORs of 1.05 (95\% CI 0.99-1.11) and 1.01 (95\% CI 0.95-1.07), respectively.

\section{ROC curve of muscle mass}

The ROC curves of muscle mass plotted for the diagnosis of NAFLD by sex are shown in Fig. 2. The cut-off value of muscle mass was $8.0 \mathrm{~kg} / \mathrm{m}^{2}$ in men and $4.9 \mathrm{~kg} /$ $\mathrm{m}^{2}$ in women, with sensitivities of 33.9 and $68.8 \%$ and specificities of 90.0 and $47.6 \%$, respectively. The areas under the ROC curve for NAFLD were 0.624 (95\% CI $0.501-0.748, P<0.063)$ and 0.592 (95\% CI 0.531-0.653, $P<0.031)$ in men and women, respectively.

\section{Discussion}

NAFLD is a spectrum of liver disease that has now become the most common cause of chronic liver disease in adults of all ethnicities. The risk of NAFLD increases with age [17]. Sarcopenia is described as involuntary loss of muscle mass, muscle strength and muscle performance that occurs with ageing, which can cause adverse health outcomes such as falls, disability, poor quality of life, and even mortality according to the AWGS. The AWGS also recommends cut-off values for muscle mass measurements $\left(7.0 \mathrm{~kg} / \mathrm{m}^{2}\right.$ for men and $5.4 \mathrm{~kg} / \mathrm{m}^{2}$ for women by using DXA), HGS $(<26 \mathrm{~kg}$ for men and $<18$ $\mathrm{kg}$ for women), and usual gait speed $(<0.8 \mathrm{~m} / \mathrm{s})$. Sarcopenia is considered a new geriatric syndrome that is 
Table 4 ORs and 95\% Cls of muscle mass and muscle strength for NAFLD

\begin{tabular}{|c|c|c|c|c|}
\hline & Men & & & \\
\hline \multirow[t]{2}{*}{ Variable } & Model 1 & & Model 2 & \\
\hline & OR $(95 \% \mathrm{Cl})$ & $P$ & OR $(95 \% \mathrm{Cl})$ & $P$ \\
\hline Muscle mass, per SD decrease & $2.91(1.58,5.35)$ & 0.001 & $2.88(1.52,5.46)$ & 0.001 \\
\hline \multirow[t]{2}{*}{ Muscle strength, per SD decrease } & $1.15(1.04,1.26)$ & 0.004 & $1.15(1.02,1.28)$ & 0.021 \\
\hline & Women & & & \\
\hline \multirow[t]{2}{*}{ Variable } & Model 1 & & Model 2 & \\
\hline & OR $(95 \% \mathrm{Cl})$ & $P$ & OR $(95 \% \mathrm{Cl})$ & $P$ \\
\hline Muscle mass, per SD decrease & $1.89(1.51,2.38)$ & $<0.001$ & $2.08(1.63,2.67)$ & $<0.001$ \\
\hline Muscle strength, per SD decrease & $1.05(0.99,1.11)$ & 0.058 & $1.01(0.95,1.07)$ & 0.716 \\
\hline
\end{tabular}

closely associated with metabolic disorders, which denotes its importance in health care for older people [18]. In this cross-sectional sample of a Chinese study, we aimed to examine the sex-specific association between NAFLD and sarcopenia with its two crucial components using hepatic imaging and DXA scans, according to the AWGS criteria. Our data indicated that men and women with NAFLD both had markedly lower muscle mass and were more likely to have lower muscle strength than controls. The status of sarcopenia and sarcopenic obesity leads to an increased prevalence of NAFLD. Moreover, LMM appeared to be a better predictor for NAFLD prevalence than LMS. Further multivariable analysis identified that participants with LMM had statistically higher odds of suffering from NAFLD than participants with LMS in both men and women.

Recent clinical studies have already demonstrated a positive relationship between sarcopenia and the prevalence of NAFLD $[6,11,19,20]$. Hong et al. first found that the OR for NAFLD risk was 5.16 (95\% CI 1.63-16.33) in the lowest quartile of SMI compared to the highest quartile [6]. Kim et al. showed that men and women with NAFLD had markedly lower HGS and were more likely to have LMS than controls [11]. However, most of these studies focused on a single component of sarcopenia, which was not sufficient to fully understand the relationship between muscle status and NAFLD. It is unclear whether LMM or LMS is independently associated with NAFLD in the same model. In the present study, we found that men and women with NAFLD had markedly lower SMIs and were more likely to have LMM than controls. LMM was associated with an increased risk of NAFLD, with ORs of 2.88 (95\% CI 1.52-5.46) in men and 2.08 (95\% CI 1.63-2.67) in women. However, participants with LMS showed a slightly higher odds of NAFLD, with an OR of 1.15 (95\% CI 1.02-1.28) in men only.

Moreover, since sarcopenia has been described as an age-associated decline in muscle mass as well as muscle function (defined by muscle strength or physical performance), it is also important to focus on the (a)

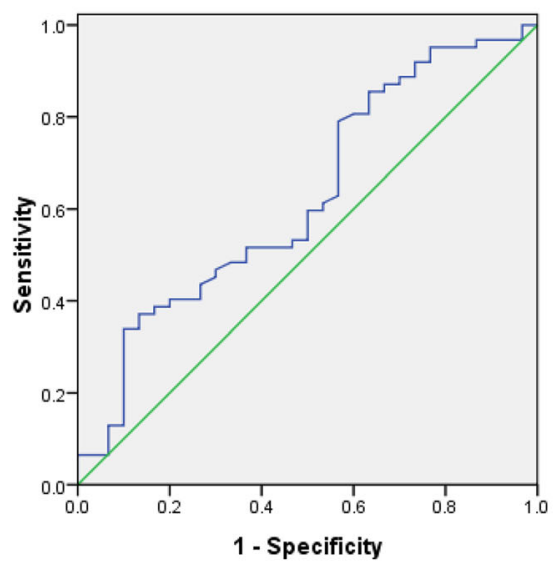

(b)

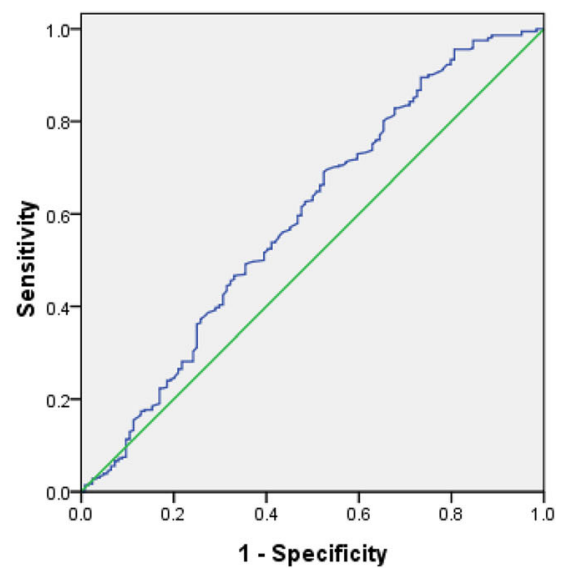

Fig. 2 ROC curves of muscle mass to predict the presence of NAFLD by sex. a Men $(A \cup C=62.4 \%, P<0.063)$; $\mathbf{b}$ Women $(A \cup C=59.2 \%, P<0.031)$ 
relationship between physical performance and NAFLD. We found that subjects with NAFLD had significantly lower GS than NAFLD-free subjects in both men and women. Further Pearson correlation coefficients showed that GS had a positive association with muscle strength (men: $r=0.315, P=0.002$, women: $r=0.538, P<0.001$ ). A wide range of tests for physical performance are recommended when diagnosing sarcopenia, including the Short Physical Performance Battery (SPPB), usual gait speed walk test, stair climb power test, and timed-upand-go test (TUG) [15]. A Taiwanese study examined the association of NASH with physical fitness, which demonstrated that men with NASH had fewer 2-min situp numbers and longer times to complete a $3000-\mathrm{m}$ run than unaffected men [21]. Exercise, such as aerobic exercise, resistance training, and their combination, can increase muscle protein synthesis and promote anabolism, which can effectively improve the progression of sarcopenia and NAFLD [22, 23]. Therefore, since both NAFLD and sarcopenia may result in reduced physical capability and poor quality of life, the status of comorbidity in older adults should be given extensive attention.

Previous studies also attempted to elucidate the mechanism of sarcopenia development in patients with NAFL D. Currently, growing attention has been given to sexspecific differences in the development of sarcopenia and NAFLD. In this study, men with LMM had an increased risk of suffering from NAFLD, with higher odds after adjustment compared to female participants. Regarding muscle strength, low HGS was associated with an increased NAFLD prevalence in men only. Yang et al. found that some metabolic syndromes may make men more prone to sarcopenia, likely due to the low levels of physical activity associated with such conditions [24]. A small cross-sectional study from Japan revealed that the SMI had a negative association with hepatic steatosis only in men with type 2 diabetes [25]. Then, a more extensive population-based study involving 4210 men and women also suggested that sarcopenia was independently associated with NAFLD in men with type 2 diabetes, while no significant difference was found in women [26]. In our study, we found that male subjects with NAFLD were younger than those without NAFLD; in contrast, female subjects with NAFLD were older than those without NAFLD. In addition to the reasons of genetic predisposition and different lifestyles, changes in basal hormone levels with age may also lead to this opposite outcome between older men and women. Some studies have confirmed that dysregulated sex hormone disorders are involved in the pathogenesis of NAFLD and sarcopenia. Testosterone, a potent anabolic hormone, can promote muscle protein synthesis [27]. Sarcopenia, which is related to the reduction in physical activity, the lack of anabolic hormones, and the decrease in proinflammatory cytokines, has been associated with NAFLD independent of metabolic syndrome features $[5,28]$. Extrapolating from these findings, the regulation of sex hormones may be involved in the mechanism of sex-specific differences in the development of sarcopenia and NAFLD.

Several epidemiological and experimental studies have shown that insulin resistance may have an essential role in sarcopenia. Skeletal muscle is recognized as a tissue that is primarily responsible for peripheral insulinmediated glucose disposal. Insulin resistance may reduce the stimulation of the protein synthesis pathway and increase the activation of the protein degradation pathway, which might eventually lead to muscle loss $[18,29]$. Indeed, the prevalence of sarcopenic obesity was higher among the participants with NAFLD in our study. Meanwhile, Pearson correlation coefficient analyses confirmed that the level of FPG was inversely associated with muscle mass in men and HGS in women. The serum level of TG was also negatively correlated with HGS in women. In this context, our current research also implied that both LMM and LMS might participate in the progression of NAFLD through dyslipidaemia and insulin resistance.

It is also worth mentioning that compared to LMS, LMM had higher odds of increasing the risk of NAFLD in our study. In women, sarcopenia was only found to have a statistically significant relationship with NAFLD when defined in terms of muscle mass alone, which was consistent with early research focused on the relationship between sarcopenia and metabolic syndrome. This might suggest that muscle mass, to some extent, was more significant than muscle strength in the context of NAFLD.

However, several limitations should also be considered when interpreting the results. First, the diagnosis of NAFL D was based on hepatic ultrasonography, and histologic confirmation of NAFLD by liver biopsy was not available. Second, the study was limited to verifying causality due to the cross-sectional design. Third, the information regarding past medical history was self-reported, which might have led to recall bias. Additionally, the number of subjects with sarcopenic obesity was low, which needs further enlargement of the sample size to allow for an adequate statistical comparison. Finally, our study population was exclusively Chinese, so the results might not be generalizable to other populations.

\section{Conclusion}

This work is among the few studies to examine the independent association of the two crucial components of sarcopenia (LMM and LMS) with NAFLD, stratified by sex according to the AWGS. LMM was consistently associated with NAFLD in both men and women, while 
LMS was associated with NAFLD only in men after adjustment for potential confounders. Moreover, compared to muscle strength, muscle mass was a better predictor for the presence of NAFLD in both sexes. Given that the understanding of the close relationship between NAFLD and sarcopenia is of great interest in this era of the ageing population, further well-designed studies should focus on common therapeutic strategies to prevent muscle wasting as well as NAFLD.

\begin{abstract}
Abbreviations
ALB: Albumin; ALT: Alanine aminotransferase; ASM: Appendicular skeletal muscle mass; AST: Aspartate aminotransferase; AWGS: Asian Working Group for Sarcopenia; Cl: Confidence interval; Cr: Creatinine; DXA: Dual-energy X-ray absorptiometry; FPG: Fasting plasma glucose; GGT: Gammaglutamyltransferase; GS: Gait speed; HDL-C: high-density lipoprotein cholesterol; HGS: Handgrip strength; LDL-C: Low-density lipoprotein cholesterol; LMM: Low muscle mass; LMS: Low muscle strength; NAFL D: Non-alcoholic fatty liver disease; NASH: Non-alcoholic steatohepatitis; OR: Odds ratio; SMI: Skeletal muscle index; TC: Total cholesterol; TG: Triglyceride
\end{abstract}

\section{Acknowledgements}

Not applicable.

\section{Authors' contributions}

YMY and YMW conceived and designed the study. DFD, YCY and WWL collected patient samples. WJZ and JX verified the data. KFZ and WJZ performed the statistical analysis under the guidance of $\mathrm{QZ}$. YMW wrote the first draft of the manuscript. KFZ and WJZ were mostly involved in several revisions of the manuscript. YMY provided critical comments and joined other authors in revising the manuscript and approving the final submission. All authors have read and approved the manuscript in its current state.

\section{Funding}

This study was funded by the Medical Health Science and Technology Project of Zhejiang Provincial Health Commission (No.2021430262 and No. 2020382690), the Project of Administration of Traditional Chinese Medicine of Zhejiang Province of China (No. 2018ZQ004), the National Key R\&D Program of China (No. 2018YFC2000301 and No. 2018YFC2001405). These funding sources had no involvement in the collection, analysis and interpretation of data; in the writing of the report; or in the decision to submit the article for publication.

\section{Availability of data and materials}

The datasets used and/or analysed during the current study are available from the corresponding author on reasonable request.

\section{Ethics approval and consent to participate}

All procedures performed in studies involving human participants were in accordance with the 1964 Helsinki declaration and its later amendments or comparable ethical standards. The research ethics committee of the First Affiliated Hospital, School of Medicine, Zhejiang University, approved the study. The reference number was 2008-1106. Written informed consent was obtained from all participants included in the study.

\section{Consent for publication}

Not Applicable.

\section{Competing interests}

The authors declare that they have no competing interests.

\section{Author details}

'Department of Geriatrics, the First Affiliated Hospital, School of Medicine, Zhejiang University, Hangzhou 310003, Zhejiang, China. ${ }^{2}$ Zhejiang Provincial Key Laboratory for Diagnosis and Treatment of Aging and Physic-chemical Injury Diseases, the First Affiliated Hospital, School of Medicine, Zhejiang University, Hangzhou 310003, Zhejiang, China. ${ }^{3}$ Department of Cardiology, Zhejiang Hospital, Hangzhou 310013, Zhejiang, China. ${ }^{4}$ Department of
Emergency Medicine, The First Affiliated Hospital, School of Medicine, Zhejiang University, Hangzhou 310003, Zhejiang, China.

Received: 1 May 2020 Accepted: 17 November 2020 Published online: 14 January 2021

\section{References}

1. Chalasani N, Younossi Z, Lavine JE, Diehl AM, Brunt EM, Cusi K, Charlton M, Sanyal AJ. The diagnosis and management of non-alcoholic fatty liver disease: practice guideline by the American Association for the Study of Liver Diseases, American College of Gastroenterology, and the American Gastroenterological Association. Hepatology. 2012;55(6):2005-23.

2. Estes C, Razavi H, Loomba R, Younossi Z, Sanyal AJ. Modeling the epidemic of nonalcoholic fatty liver disease demonstrates an exponential increase in burden of disease. Hepatology. 2018;67(1):123-33.

3. Golabi P, Otgonsuren M, de Avila L, Sayiner M, Rafiq N, Younossi ZM. Components of metabolic syndrome increase the risk of mortality in nonalcoholic fatty liver disease (NAFLD). Medicine (Baltimore). 2018; 97(13):e0214.

4. Rinella ME, Sanyal AJ. Management of NAFLD. A stage-based approach. Nat Rev Gastroenterol Hepatol. 2016;13(4):196-205.

5. Bhanji RA, Narayanan P, Allen AM, Malhi H, Watt KD. Sarcopenia in hiding: the risk and consequence of underestimating muscle dysfunction in nonalcoholic steatohepatitis. Hepatology. 2017;66(6):2055-65.

6. Hong HC, Hwang SY, Choi HY, Yoo HJ, Seo JA, Kim SG, Kim NH, Baik SH, Choi DS, Choi KM. Relationship between sarcopenia and nonalcoholic fatty liver disease: the Korean Sarcopenic obesity study. Hepatology. 2014;59(5): 1772-8.

7. Carias S, Castellanos AL, Vilchez V, Nair R, Dela Cruz AC, Watkins J, Barrett T, Trushar P, Esser K, Gedaly R. Nonalcoholic steatohepatitis is strongly associated with sarcopenic obesity in patients with cirrhosis undergoing liver transplant evaluation. J Gastroenterol Hepatol. 2016;31(3):628-33.

8. Bhanji RA, Carey EJ, Yang L, Watt KD. The long winding road to transplant: how sarcopenia and debility impact morbidity and mortality on the waitlist. Clin Gastroenterol Hepatol. 2017;15(10):1492-7.

9. Sanchez-Rodriguez D, Marco E, Cruz-Jentoft AJ. Defining sarcopenia: some caveats and challenges. Curr Opin Clin Nutr Metab Care. 2020;23(2):127-32.

10. Kim G, Lee $S E$, Lee YB, Jun JE, Ahn J, Bae JC, Jin SM, Hur KY, Jee JH, Lee MK, et al. Relationship between relative skeletal muscle mass and nonalcoholic fatty liver disease: a 7-year longitudinal study. Hepatology. 2018:68(5):1755-68.

11. Kim BJ, Ahn SH, Lee SH, Hong S, Hamrick MW, Isales CM, Koh JM Lower hand grip strength in older adults with non-alcoholic fatty liver disease: a nationwide population-based study. Aging (Albany NY). 2019; 11(13):4547-60

12. Cruz-Jentoft AJ, Baeyens JP, Bauer JM, Boirie Y, Cederholm T, Landi F, Martin FC, Michel JP, Rolland Y, Schneider SM, et al. Sarcopenia: European consensus on definition and diagnosis: report of the European working group on sarcopenia in older people. Age Ageing. 2010;39(4):412-23.

13. Studenski SA, Peters KW, Alley DE, Cawthon PM, McLean RR, Harris TB, Ferrucci L, Guralnik JM, Fragala MS, Kenny AM, et al. The FNIH sarcopenia project: rationale, study description, conference recommendations, and final estimates. J Gerontol A Biol Sci Med Sci. 2014;69(5):547-58.

14. Su W, Cao H, Fan J. Diagnosis and treatment of non-alcoholic fatty liver disease. Zhonghua Gan Zang Bing Za Zhi. 2014;22(9):705-7.

15. Chen LK, Liu LK, Woo J, Assantachai P, Auyeung TW, Bahyah KS, Chou MY, Chen LY, Hsu PS, Krairit O, et al. Sarcopenia in Asia: consensus report of the Asian working Group for Sarcopenia. J Am Med Dir Assoc. 2014;15(2):95-101.

16. Fan JG, Jia JD, Li YM, Wang BY, Lu LG, Shi JP, Chan LY. Chinese Association for the Study of liver D: guidelines for the diagnosis and management of nonalcoholic fatty liver disease: update 2010: (published in Chinese on Chinese journal of Hepatology 2010; 18:163-166). J Dig Dis. 2011;12(1):38-44.

17. Hamaguchi M, Kojima T, Ohbora A, Takeda N, Fukui M, Kato T. Aging is a risk factor of nonalcoholic fatty liver disease in premenopausal women. World J Gastroenterol. 2012;18(3):237-43.

18. Kalyani RR, Corriere M, Ferrucci L. Age-related and disease-related muscle loss: the effect of diabetes, obesity, and other diseases. Lancet Diabetes Endocrinol. 2014;2(10):819-29.

19. Alferink LIM, Trajanoska K, Erler NS, Schoufour JD, de Knegt RJ, Ikram MA, Janssen HLA, Franco OH, Metselaar HJ, Rivadeneira F, et al. Nonalcoholic 
fatty liver disease in the Rotterdam study: about muscle mass, sarcopenia, fat mass, and fat distribution. J Bone Miner Res. 2019;34(7):1254-63.

20. Koo BK, Kim D, Joo SK, Kim JH, Chang MS, Kim BG, Lee KL, Kim W. Sarcopenia is an independent risk factor for non-alcoholic steatohepatitis and significant fibrosis. J Hepatol. 2017;66(1):123-31.

21. Chen YJ, Chen KW, Shih YL, Su FY, Lin YP, Meng FC, Lin F, Yu YS, Han CL, Wang $\mathrm{CH}$, et al. Chronic hepatitis $\mathrm{B}$, nonalcoholic steatohepatitis and physical fitness of military males: CHIEF study. World J Gastroenterol. 2017; 23(25):4587-94.

22. Austin P, Gerber L, Paik JM, Price JK, Escheik C, Younossi ZM. Aerobic capacity and exercise performance in nonalcoholic fatty liver disease. J Sports Med Phys Fitness. 2019;59(8):1376-88.

23. Batsis JA, Villareal DT. Sarcopenic obesity in older adults: aetiology, epidemiology and treatment strategies. Nat Rev Endocrinol. 2018;14(9):513-37.

24. Yang L, Smith L, Hamer M. Gender-specific risk factors for incident sarcopenia: 8-year follow-up of the English longitudinal study of ageing. J Epidemiol Community Health. 2019;73(1):86-8.

25. Hashimoto Y, Osaka T, Fukuda T, Tanaka M, Yamazaki M, Fukui M. The relationship between hepatic steatosis and skeletal muscle mass index in men with type 2 diabetes. Endocr J. 2016;63(10):877-84.

26. Seo DH, Lee YH, Park SW, Choi YJ, Huh BW, Lee E, Huh KB, Kim SH, Cha BS Sarcopenia is associated with non-alcoholic fatty liver disease in men with type 2 diabetes. Diabetes Metab. 2020;46(5):362-69.

27. Yim JY, Kim J, Kim D, Ahmed A. Serum testosterone and non-alcoholic fatty liver disease in men and women in the US. Liver Int. 2018;38(11):2051-9.

28. Lee YH, Jung KS, Kim SU, Yoon HJ, Yun YJ, Lee BW, Kang ES, Han KH, Lee HC, Cha BS. Sarcopaenia is associated with NAFLD independently of obesity and insulin resistance: Nationwide surveys (KNHANES 2008-2011). J Hepatol. 2015;63(2):486-93.

29. Wannamethee SG, Atkins JL. Muscle loss and obesity: the health implications of sarcopenia and sarcopenic obesity. Proc Nutr Soc. 2015;74(4): 405-12.

\section{Publisher's Note}

Springer Nature remains neutral with regard to jurisdictional claims in published maps and institutional affiliations.

Ready to submit your research? Choose BMC and benefit from:

- fast, convenient online submission

- thorough peer review by experienced researchers in your field

- rapid publication on acceptance

- support for research data, including large and complex data types

- gold Open Access which fosters wider collaboration and increased citations

- maximum visibility for your research: over $100 \mathrm{M}$ website views per year

At $\mathrm{BMC}$, research is always in progress.

Learn more biomedcentral.com/submissions 